\title{
Risikomanagement verstärken
}

\section{Bruno Brühwiler}

Geschäftsführer Euro Risk Limited, Zürich

Experte in Working Group ISO 31000 Risk Management, Projektleiter ONR 49000

\footnotetext{
* Der erste Beitrag mit dem Titel «Klinisches Risikomanagement in der Schweiz - Bestandesaufnahme» ist in Ausgabe 1-2/2010 der SÄZ erschienen. www.saez.ch $\rightarrow$ Archiv $\rightarrow 2010 \rightarrow 1$
}

\section{Elemente des Risikomanagements}

Das Risikomanagement in einem Spital ist einerseits geprägt durch die verschiedenen Instrumente der Risikobeurteilung und Risikobewältigung, andererseits durch den organisatorischen Rahmen bzw. durch den Auftrag und die Verpflichtung der Führung, ein Risikomanagement einzuführen, umzusetzen, zu bewerten und laufend zu verbessern. Zwei Fragen stehen dabei im Zentrum des Interesses: 1. Worauf soll sich das Risikomanagement in einem Spital materiell fokussieren? 2. Wie kann man Risikomanagement betreiben, ohne (erneut) eine Bürokratie einzurichten, für die man bekanntlich keine Ressourcen freilegen kann? Viele Risikomanagement-Massnahmen sind schon vorhanden, es geht jetzt darum, diese zu verstärken, zu systematisieren und ihre Umsetzung einzufordern.

\section{Die Szenario-Analyse \\ für die Schwerpunktbildung}

Eine der bekannten Instrumente des Risikomanagements ist die sogenannte Szenario-Analyse. Sie zeichnet ein Risikoprofil - in unserem Fall betreffend die Gefährdung der Patientensicherheit - mit den rund zehn Hauptrisiken, die individuell im Spital zu Handlungsbedarf führen. Dabei setzen sich die Risikoeigner in einem Team zusammen, um die wichtigen Risiken für die Patientensicherheit zu identifizieren, zu analysieren, zu bewerten und Massnahmen der Risikominderung zu entwickeln. Das Risikoprofil könnte für ein Krankenhaus konkret wie folgt aussehen:

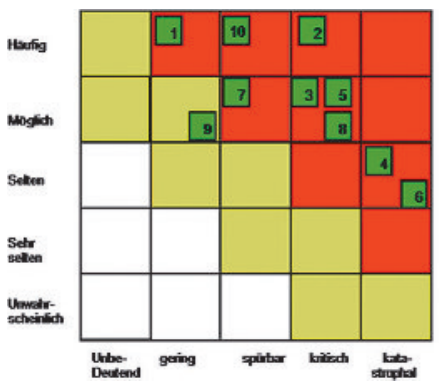

\begin{tabular}{|c|l|}
\hline 1 & Sturz und Verletzung von Patienten \\
\hline 2 & Informationsverlust Dienstūbergaben \\
\hline 3 & Verwechslung von Patienten oder Seiten \\
\hline 4 & Falsche Verabreichung von Arzneimitteln \\
\hline 5 & Fehldiagnose und Fehlbehandlung \\
\hline 6 & Nosokomiale Infektion \\
\hline 7 & Fehlbedienung von Medizinprodukten \\
\hline 8 & Entlassungsmanagement \\
\hline 9 & Lūcken in der Patientenaufilärung \\
\hline 10 & Informations- und Betriebsicherheit der IT \\
\hline
\end{tabular}

Die 10 Hauptrisiken eines Spitals.

Korrespondenz: Dr. Bruno Brühwiler Euro Risk Limited Talstrasse 82 CH-8022 Zürich

bruno.bruehwiler@eurorisk.ch
Mit den zehn in der Abbildung aufgeführten Punkten sind beispielhaft die Hauptrisiken eines Spitals aufgezeigt. Es geht im Risikomanagement nun darum, sehr konzentriert in diesen definierten Risikofeldern mit individuell festgelegten Prioritäten an der Verbesserung der Patientensicherheit zu arbeiten. Wenn ein

\section{Renforcer la gestion des risques}

L'organisation de la gestion des risques dans un hôpital se base sur deux considérations essentielles: (1) Sur quoi la gestion des risques (et/ou la gestion de la qualité) doit-elle se fonder sur le plan matériel? (2) Comment peut-on pratiquer une gestion des risques efficace sans bureaucratie supplémentaire? Des méthodes relativement simples (alliées au système de gestion des incidents critiques CIRS) servent d'introduction à cette thématique.

L'analyse des risques basée sur un scénario cherche à définir les principales zones de risques d'un hôpital. II doit également servir à jeter un pont entre le système CIRS en place et les informations recensées en matière de risque. Cela permet de garantir que les priorités et les ressources se concentrent sur l'essentiel.

L'analyse du risque sert à la gestion des processus. La préoccupation fondamentale de la gestion de la qualité est systématiquement axée sur les risques pour la patientèle, inhérents aux processus. Si toutes les personnes impliquées sont conscientes des risques, elles développent une attention particulière pour les points qui pourraient fréquemment mal tourner.

La gestion des risques fait partie du mandat et des devoirs des étages supérieurs de la direction. La politique de gestion des risques doit rendre visible la manière dont l'hôpital souhaite déterminer et traiter les risques pour les patients (de même que les autres risques). L'audit du système de gestion des risques doit montrer l'efficacité des mesures prises pour la sécurité des patients. Les nouvelles normes internationales ISO 31000 et leur mise en application selon les règles de la série ONR 49000 peuvent aider concrètement à y parvenir. 
Spital dieses individuelle Risikoprofil kennt und seine Handlungspakete priorisiert, lassen sich die Ressourcen auf die wesentlichen Themen ausrichten.

\section{Brückenschlag zum CIRS}

Es besteht zwischen dem Risikoprofil und dem Critical Incidents Reporting System (CIRS) eine direkte Verbindung. Das CIRS liefert detaillierte Risikoinformationen über viele Vorfälle, die in einzelnen Abteilungen des Spitals festgestellt wurden. Diese Informationen sind Risikoindikatoren und können dazu genutzt werden, die Risikoidentifikation und die Risikoeinschätzung in den Risikoprofilen zu unterstützen. Oft fehlen nämlich gerade individuell nutzbare oder anerkannte statistische Daten. Öffentlich zugängliche Mortalitätsstatistiken wie z.B. diejenige des BAG lassen sich zwar nutzen, tragen aber nichts zu Risikobewältigung bei.
Mitarbeitenden, die Risiken besser zu beherrschen. Ein Ergebnis der Prozessgestaltung besteht in der Einführung einer Operations-Checkliste.

Eine in acht Ländern durchgeführte Studie zeigt, dass durch die konsequente Anwendung einer Checkliste vor dem chirurgischen Eingriff sowohl die Mortalitätsraten als auch die Komplikationen etwa halbiert werden konnten [1]. «Die Spitäler waren angehalten, zunächst rund 500 Operationen wie gewohnt vorzunehmen. Im zweiten Teil der Untersuchung erhielten sie dann die Vorgabe, bei weiteren 500 Eingriffen die Checkliste der WHO zu verwenden. Die darin aufgeführten 19 Sicherheitsfragen sollen unter anderem sicherstellen, dass die richtige Operation beim richtigen Patienten erfolgt, alle notwendigen diagnostischen und therapeutischen Massnahmen Berücksichtigung finden und vorhersehbare Komplikationen

\section{CIRS allein liefert viele Detailinformationen mit der Gefahr, vor lauter Bäumen den Wald nicht mehr zu sehen}

Im Gegensatz zum CIRS und zum individuellen Risikoprofil helfen sie nicht, die Risiken und deren Ursachen zu verstehen und damit die Risiken zu beseitigen oder einzudämmen.

CIRS und Risikoprofil ergänzen sich. CIRS allein liefert viele Detailinformationen mit der Gefahr, vor lauter Bäumen den Wald nicht mehr zu sehen. Das Risikoprofil hingegen verdichtet die Risikoinformationen und ermöglicht es dem Spital und seiner Leitung, ohne tagesindividuelle Ablenkung an den entscheidenden Risikoschwerpunkten zu arbeiten. Vertieftes Risikomanagement muss in der Folge auch zur Prozessgestaltung führen, für die die Gefährdungsanalyse ein weiteres, scharfes Instrument zur Verfügung stellt.

\section{Gefährdungsanalyse und Prozessgestaltung}

Die Gefährdungsanalyse (oft auch mit der FMEA «Failure Mode and Effects Analysis» oder «Fehlermöglichkeiten und Einflussanalyse» gleichgesetzt) ist ein in der Industrie und auch in anderen Bereichen bekanntes und weitverbreitetes Instrument. Besonders die Automobilindustrie verdankt ihr grosse Erfolge in der Serienqualität. Natürlich sind die Patienten nicht mit Autos zu vergleichen. Aber ein Spital hat eine «Produktionsfunktion», die ebenfalls möglichst fehlerfrei oder fehlerarm sein soll.

Die Gefährdungsanalyse setzt dort an, wo das Qualitätsmanagement seinen Ausgangspunkt haben sollte: In der Prozessanalyse und Prozessgestaltung. Die Aufgabe besteht nun darin, die medizinischen Prozesse auf ihre Risiken hin zu analysieren und die Risiken durch gezielte Massnahmen der Prozessgestaltung, unter Einbindung aller am Prozess beteiligten wie Allergien bedacht werden. Empfohlen wird zudem, nach dem Eingriff das Operationsmaterial nachzuzählen. Denn es kommt immer wieder vor, dass Werkzeuge oder Tücher im Körper des Patienten zurückbleiben, wo sie schwere Infektionen hervorrufen können» [2].

Allerdings muss darauf hingewiesen werden, dass der Einsatz von Checklisten zwar sinnvoll sein kann, aber in einem Krankenhaus nicht zum Wundermittel erklärt werden sollte. Die Menschen müssen miteinander auch «normal», d.h. ohne Checklisten arbeiten können. Aber wo eine Checkliste eingeführt wird, sollte man ihre konsequente Anwendung einfordern. Dies muss so weit gehen, dass ein chirurgischer Eingriff nicht ausgeführt werden darf, wenn die Checkliste nicht vollständig ausgefüllt und auch paraphiert ist. Notfallsituationen sind dabei selbstverständlich speziell zu behandeln.

\section{Offene Fehler- und Risikokultur}

Wirksames Risikomanagement erfordert eine offene Fehler- bzw. Risikokultur. Hinter diesen Worten verbergen sich nicht etwa Ideale oder Glaubensbekenntnisse. Eine offene Risikokultur ist in der bereits erwähnten ONR 49000 wie folgt definiert: «Denken, Handeln und Verhalten einer Organisation nach den Regeln und Grundsätzen des Risikomanagements» [3]. Im Spital ist es ganz wichtig, dass die in den Behandlungsprozessen eingebundenen Ärzte, Schwestern und weitere Personen offen über Risiken und Vorkommnisse sprechen können, ohne Angst vor Schuld und Strafe. Eine Risikokultur kann also nur entstehen, wenn es die Organisation mit dem Risikomanagement ernst meint und die Risikoinformationen konsequent 
dazu nutzt, die Patientensicherheit zu verbessern. Voraussetzung dafür ist der Auftrag und die Verpflichtung der obersten Leitung.

\section{Auftrag und Verpflichtung der Führung}

Im Krankenhaus ist davon auszugehen, dass die Leitung auf allen Ebenen das grösste Interesse hat, die Patientensicherheit zu gewährleisten und alle Massnahmen, die mit den vorhandenen Ressourcen für die Förderung der Patientensicherheit umsetzbar sind, einzuleiten und zu unterstützen.

Ein aktives Risikomanagement stellt sich aber nicht von selbst ein, sondern wird dadurch ermöglicht, dass es von der Führung initialisiert und thematisiert wird. Dies bedingt nicht nur ein grundsätzliches Bekenntnis, sondern auch konkrete Aussagen darüber, wie Risikomanagement praktisch durchgeführt werden soll. Die Einrichtung eines CIRS ist ein guter Anfang. Die Identifikation der wirklich zentralen, für das Spital spezifischen Risikomanagement-Themen mit dem Szenario-Risikoprofil ist ein weiterer Schritt. Schliesslich geht es darum, daraus wohlüberlegte Massnahmenpakete abzuleiten und auf der Zeitachse mit den erforderlichen Ressourcen umzusetzen. Oft geht es bei der Realisierung eines wirksamen Risikomanagements nicht um zusätzliches Geld, sondern um gute medizinische Arbeit und um gute Kommunikation und Führung. Allerdings braucht es dazu Führungskräfte und Fachleute, die Methoden und Prozesse im Risikomanagement kennen und konkret implementieren können.

Wenn die Führung eines Spitals eine klare Vorstellung über die Möglichkeiten und die Instrumentierung des Risikomanagements erarbeitet hat, ist es zweckmässig, diese in einer Risikomanagement-Politik schriftlich zu formulieren. Ein solches Statement kann in drei Teile gegliedert werden mit folgenden Inhalten:

- Grundsätze für das Risikomanagement: Darin werden die Ziele, Anwendungen und Verantwortlichkeiten und der Führungsregelkreis (planen, umsetzen, bewerten und verbessern) für das Risikomanagement definiert.

- Umsetzung des Risikomanagements: Hier sind die Instrumente und ihr Einsatz beschrieben, z.B. CIRS, die Szenarioanalyse und die Prozessgestaltung für eine sichere Patientenbehandlung.

- Integration des Risikomanagements: Schliesslich soll die Risikomanagement-Politik sich auch dazu äussern, wie das Risikomanagement in der Organisation eingebettet ist und wie es sich zu andern Tätigkeiten, z.B. dem Qualitätsmanagement und dem Schadensmanagement (Versicherung!) verhält.

Die oberste Leitung eines Spitals sollte z. B. am Schluss eines Geschäftsjahres auch einen Risikomanagement-
Bericht erstellen lassen, in dem dargestellt ist, ob das Risikomanagement wirksam ist und wie sich die Risiken manifestiert und allenfalls (positiv oder negativ) verändert haben.

\section{Fähigkeiten, mit Risiken aktiv umzugehen}

In den meisten Spitälern der Schweiz sind Risikomanagement-Tätigkeiten etabliert. Es geht darum, dass diese aktiviert und systematisch weiterentwickelt werden, unter gezielter Nutzung der vorhandenen Ressourcen. Der Schlüssel des Risikomanagements liegt in der Fähigkeit von Menschen und Teams, mit Risiken aktiv umzugehen. Diese Menschen werden im Risikomanagement als «Risikoeigner» und «Risikomanager» bezeichnet. Sie sind heute in den meisten Spitälern der Schweiz schon da, man muss sie nur richtig ausbilden und ihre Tätigkeit sinnvoll auf das Anliegen der Patientensicherheit ausrichten. Die neuen Standards ISO 31000 Risk Management und ONR 49000 Risikomanagement von Organisationen und Systemen unterstützen dabei die Spitäler und ihre Führung.

Die ONR 49003 befasst sich mit der Ausbildung und Qualifikation des Risikomanagers, mit Anwendung auch im klinischen Risikomanagement. Der Risikomanager muss in der Lage sein, Risikobeurteilungen zu erstellen, Massnahmen für die Risikoverbesserung auszuarbeiten und deren Umsetzung konkret zu unterstützen. Dazu gehört auch die Fähigkeit, die Verbindung zwischen Managementsystem und Risikomanagement herzustellen und die Synergien auszuschöpfen. Er sollte einige wichtige Methoden der Risikobeurteilung wie die Szenarioanalyse, die Gefährdungsanalyse sowie deren Zusammenspiel mit dem CIRS nicht nur verstehen, sondern konkret umsetzen können.

Wichtig ist dabei, dass der Risikomanager, zusammen mit dem Risikoeigner, an den wichtigen Risiken der Patientensicherheit arbeitet. Deshalb sind die guten Risikomanager Ärzte, Schwestern und Pfleger sowie andere Mitarbeitende, die z.B. im Bereich der medizinischen und technischen Infrastruktur tätig sind. Risikomanagement ist schliesslich keine Bürokratie, sondern zuverlässige, risikobewusste Arbeit am oder beim Patienten.

\section{Literatur}

1 Haynes AB et al. A Surgical Safety Checklist to Reduce Morbidity and Mortality in a Global Population. NEJM. 2009;(360):491-9.

2 Vgl. NZZ. www.nzzglobal.ch/nzz/forms/page.htm vom 21. Januar 2009.

3 ONR 49 000:2008 Risikomanagement für Organisationen und Systeme. Ziff. 3.2.22. 